\title{
Évolution à court terme de formes du phosphore d'un sol ferrallitique après apports d'une boue urbaine
}

\author{
M Brossard 1, M Mench 3, M Clairon 2, JY Laurent 1 \\ avec la collaboration technique de D Nagou ${ }^{2}, \mathrm{~J}_{\text {Louri }}{ }^{1}$ \\ ' ORSTOM, laboratoire matière organique des sols tropicaux, BP 8006, F-97259 Fort-de-France Cedex; \\ 2 INRA, station agro-pédoclimatique, BP 1232, F-97184 Pointe-à-Pitre Cedex; \\ 3 INRA, Station d'agronomie, BP 81, F-33883, Villenave d'Ornon Cedex, France
}

(Reçu le 20 mars 1990; accepté le 10 juin 1991)

\begin{abstract}
Résumé - Une boue urbaine a été incorporée aux doses de 10 et $100 \mathrm{t}(\mathrm{MS}) \cdot{ }^{-1} \mathrm{a}^{-1}$ dans l'horizon $0-20 \mathrm{~cm}$ d'un sol ferrallitique de Guadeloupe. L'expérimentation est conduite en microparcelles de plein champ pendant $500 \mathrm{j}$. Après prélèvement des échantillons de sol, les mottes de boue non fragmentées de taille supérieure à $5 \mathrm{~mm}$ sont triées manuellement. Les formes du phosphore du sol analysées sont les suivantes : phosphore total $\left(P_{t}\right)$, organique $\left(P_{o}\right)$, minéral $\left(\mathrm{P}_{\mathrm{m}}\right)$, extrait par un mélange bicarbonate-fluorure $\left(\mathrm{P}_{\mathrm{NaHCO}_{3}-\mathrm{F}}\right)$. $\mathrm{A}$ la dose $10 \mathrm{t}$, les mottes de boue ne sont plus observables au-delà de $110 \mathrm{j}$ et la teneur en $\mathrm{P}_{\mathrm{t}}$ du sol augmente significativement 3 mois après enfouissement : elle est donc alors comprise entre 600 et $650 \mu \mathrm{g}^{\circ} \mathrm{g}^{-1}$. À la dose $100 \mathrm{t}$, des mottes de boue sont encore présentes après $500 \mathrm{j}$. La teneur en phosphore total du sol a triplé $\left(1400 \mu \mathrm{g} \cdot \mathrm{g}^{-1}\right)$. La cinétique d'évolution de différentes formes du phosphore montre qu'avec une dose de 10 toha ${ }^{-1}$ les formes minérales $\left(\mathrm{P}_{\mathrm{NaHCO}_{3}-\mathrm{F}}\right.$ et $\left.\mathrm{P}_{\mathrm{m}}\right)$ sont modifiées par l'apport. La teneur en phosphore organique a augmenté de $8 \%$ en fin d'expérience. Á la dose de $100 \mathrm{t}$, les teneurs des formes du phosphore analysées sont triplées par rapport au témoin : la teneur en phosphore extrait par un mélange bicarbonate-fluorure croît durant les 50 premiers j après l'épandage, puis reste supérieure à $300 \mu \mathrm{g} \cdot \mathrm{g}^{-1}$ jusqu'à $500 \mathrm{j}$. Ainsi, pour le phosphore, la dose $100 \mathrm{t}$ pourrait se justifier pour amender des sols cultivés fortement dégradés. La dose $10 \mathrm{t}$, plus compatible avec des cultures à cycles courts, accroît significativement la teneur du sol en phosphore soluble dans le mélange bicarbonate-fluorure.
\end{abstract}

phosphore total / phosphore organique / phosphore minéral / phosphore extrait bicarbonate-fluorure / zone tropicale humide

Summary - Effect of sewage sludge on short-term evolution of phosphorus forms in an oxisol. Two levels of sewage sludge (10 and 100 tons DM ha-1) were mixed in the $0-20 \mathrm{~cm}$ layer of an oxisol in Guadeloupe (French West Indies) (table l). A field experiemnt was conducted with microplots $\left(20 \mathrm{~m}^{2}\right)$ during a 500-d test period: control without sewage sludge (T), (10 T) and (100 T). After soil sampling, sewage sludge clods over $5 \mathrm{~mm}$ diameter were handsorted from soil samples. The evolution of various soil phosphorus forms total $\left(P_{t}\right)$, organic $\left(P_{o}\right)$, mineral $\left(P_{m}\right)$ and extracted with bicarbonate-fluorure $\left(P_{\mathrm{NaHCO}_{3}-\mathrm{F}}\right)$ was studied. In the $10 \mathrm{t}$ treatment sludge clods disappeared within $110 \mathrm{~d}$, and the total soil phosphorus content significantly increased 3 months after burying up to $600-650 \mu g^{\circ} \mathrm{g}^{-1}$. In the $100 \mathrm{t}$ treatment, sewage clods were still observed after $500 \mathrm{~d}$, and total soil phosphorus showed a 3-fold increase $\left(1400 \mu g^{\circ} g^{-1}\right.$ ) (figs 1 , 2). Kinetics of phosphorus forms showed that: $i$ ) the application of 10 toha ${ }^{-1}$ increased mineral phosphorus and bicarbonate-fluorure extract, organic phosphorus content showed a lower increase (fig 3); ii) 100 toha-1 had the same qualitative effects, but the various phosphorus forms increased 3-fold with respect to the control; phosphorus in the bicarbonate-fluoride extract rapidly increased and remained over $300 \mu g^{\circ} g^{-1}$ up to $500 \mathrm{~d}$ (fig 4). Application of 100 tha ${ }^{-1}$ of sewage sludge may be recommended to build up phosphorus reserves in highly degraded soils. An input of 10 t.ha- ${ }^{-1}$ seems to be sufficient to fertilize short cycle crops as it significantly increased phosphorus of the bicarbonate-fluoride extract.

total phosphorus / organic phosphorus / mineral phosphorus / bicarbonate-fluoride phosphorus / humid tropical area 


\section{INTRODUCTION}

Les teneurs élevées en matière organique, azote minéral, phosphore et oligoéléments des boues urbaines des stations d'épuration ont suscité, il y a une vingtaine d'années, leur utilisation en milieu agricole. Ce type d'amendement a pour objectif d'accroître ou de maintenir la productivité des sols à court et à long terme, et de réaliser des économies de fumures azotées et phosphatées. Toutefois, ces options doivent être réalisées en évitant la contamination du milieu par les métaux lourds ou les nitrates, qui peuvent atteindre de fortes concentrations dans ce substrat.

En Guadeloupe, les stations d'épuration produisent des boues aérobies peu chargées en micropolluants (Mench et al, 1989). Aussi, des essais de longue durée ont été mis en place par I'INRA afin de tester l'effet d'apports de boues sur un sol d'alluvions ferrallitisées du Nord-Est de la Basse Terre. La production moyenne durant 3 ans de maïs grain (var Eto amarillo) a été de $4,8 \pm 0,5 \mathrm{t} \cdot \mathrm{ha}^{-1} \cdot \mathrm{an}^{-1}$ pour un apport de $10 \mathrm{t}$ (MS) de boues ha-1 $\cdot a n^{-1}$, et de 3,6 $\pm 0,6$ t.ha 1. an ${ }^{-1}$ avec une quantité d'engrais minéraux équivalente à $N / 2$, $P$ et $K$ contenus dans un ap-

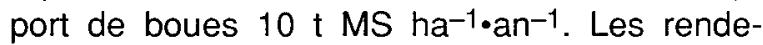
ments en grain les plus élevés $\left(7,6 \pm 0,3 \mathrm{t} \cdot h \mathrm{a}^{-1}\right)$ ont été obtenus sur les parcelles ayant reçu des boues à la dose $100 \mathrm{t}^{\text {h ha }}{ }^{-1}$ de MS (Nagou et Clairon, 1985). Substitué à la fertilisation minérale par des agriculteurs, cet amendement induit également des gains de rendement en maraîchage et bananeraie.

Les apports de boues urbaines augmentent toujours la teneur en phosphore total dans les horizons de surface des sols (King et Morris, 1972; Hinesly et al, 1973), et celle en phosphore assimilable (Morel, 1978). Dans les régions tropicales, où les sols sont caractérisés par de faibles teneurs en phosphore assimilable (Dabin, 1963; Fox et al, 1968; Chaminade et al, 1977; Roche et al, 1980; Chevignard et al, 1986) ce type d'amendement peut être, dans certains cas, une alternative à l'utilisation d'engrais minéraux. L'augmentation de la teneur en matière organique et en phosphore dans les parcelles amendées avec de fortes doses de boues a été observée dans des dispositifs de longue durée sur sol ferrallitique de Guadeloupe (Clairon et al, 1989). II est cependant nécessaire d'évaluer les quantités d'éléments nutritifs libérés par les boues. Or, à notre connaissance, il n'existe pas de données disponibles dans des conditions tropicales hu- mides. Aussi, une expérimentation en microparcelles de plein champ a été mise en place afin de préciser les transferts à court terme de matière organique et d'élements minéraux des boues vers le sol (Mench et al, 1989). Les conséquences à court terme de l'apport des boues sur l'évolution de 4 formes du phosphore du sol (total, organique, minéral, extractible par le mélange bicarbonate-fluorure) sont décrites.

\section{MATÉRIEL ET MÉTHODES}

\section{Le sol}

Dans la classification française (CPCS, 1967) il s'agit d'un sol ferrallitique faiblement désaturé, remanié, hydromorphe sur matériau alluvial argileux de Basse Terre de Guadeloupe (Domaine Duclos). Les principales caractéristiques de l'horizon $0-20 \mathrm{~cm}$ sont résumées dans le tableau 1.

\section{Dispositif expérimental}

II comprend 3 parcelles contiguës de $20 \mathrm{~m}^{2}$ : une parcelle témoin sans apports $(T)$, et 2 parcelles $(10 \mathrm{t})$ et

Tableau I. Caractéristiques générales initiales des horizons $0-20 \mathrm{~cm}$ des 3 parcelles : témoin, $[10 \mathrm{t}]$ et [100 t]. $\mathrm{P}_{\mathrm{NaHCO}_{3}}-\mathrm{F}$ : phosphore extrait dans $0,5 \mathrm{M} \mathrm{NaHCO}_{3}$ $+0,5 \mathrm{MNH}_{4} \mathrm{~F}$.

\begin{tabular}{|c|c|c|c|}
\hline & Témoin & {$[10 \mathrm{t}]$} & {$[100 t]$} \\
\hline \multicolumn{4}{|l|}{ Analyse mécanique \% } \\
\hline $\begin{array}{l}\text { Argile } \\
\text { Limons fins }\end{array}$ & 50,3 & $\begin{array}{l}50,3 \\
265\end{array}$ & $\begin{array}{l}49,2 \\
26,9\end{array}$ \\
\hline Limons grossiers & $\begin{array}{r}23,4 \\
8,0\end{array}$ & $\begin{array}{r}20,3 \\
7,3\end{array}$ & $\begin{array}{r}20,9 \\
8,0\end{array}$ \\
\hline Sables fins & 5,3 & 5,6 & 6,3 \\
\hline Sables grossiers & 7,6 & 7,3 & 6,4 \\
\hline Matière organique $\%$ & 3,8 & 3,5 & 3,5 \\
\hline $\mathrm{N}$ & 0,2 & 0,19 & 0,21 \\
\hline $\mathrm{C} / \mathrm{N}$ & 10,8 & 10,8 & 9,8 \\
\hline pH eau & 5,7 & 5,6 & 5,4 \\
\hline $\mathrm{pH} \mathrm{KCl}$ & 4,9 & 4,8 & 4,6 \\
\hline \multicolumn{4}{|l|}{ Phosphore $\mu \mathrm{gP} . \mathrm{g}^{-1}$ sol } \\
\hline total & 538 & 470 & 409 \\
\hline Olsen & 9 & 5 & 5 \\
\hline $\mathrm{PNaHCO}_{3}-\mathrm{F}$ & 31 & 28 & 34 \\
\hline CEC cmol. $\mathrm{kg}^{-1}$ & 14,8 & 14,1 & 14,6 \\
\hline \multicolumn{4}{|l|}{ Cations éch. cmol. $\mathrm{kg}^{-1}$} \\
\hline $\mathrm{Ca}^{2+}$ & 7,9 & 7,2 & 6,7 \\
\hline $\mathrm{Mg}^{2+}$ & 1,2 & 1,4 & 1,6 \\
\hline $\mathrm{K}^{+}$ & 0,2 & 0,2 & 0,3 \\
\hline $\mathrm{Na}^{+}$ & 0,1 & 0,1 & 0,1 \\
\hline $\mathrm{Al}^{3+}$ & 0,2 & 0,3 & 0,4 \\
\hline
\end{tabular}


(100 t) avec des apports respectivement de 10 et de 100 tonnes de matière sèche de boues résiduaires par ha. Le devenir des boues est suivi pendant $500 \mathrm{j}$. Durant cette période la pluviomé-trie cumulée a été de 4 $718 \mathrm{~mm}$, et la température moyenne de l'air a varié entre 22 et $27^{\circ} \mathrm{C}$. Entre l'épandage et l'enfouissement des boues la pluviomé-trie a été de $81 \mathrm{~mm}$, dont une pluie de $34 \mathrm{~mm}$. Les parcelles sont maintenues sans végétation par des désherbages manuels fréquents.

La boue urbaine (tableau II) provient de la station d'épuration de Jarry (Pointe-à-Pitre, Guadelupe). Elle est obtenue par le brassage aérobie en bassin des eaux usées, suivi d'une décantation. Les boues sont ensuite floculées par un adjuvant organique (polymère aminé, sans calcium ni fer), puis pressées (filtre à bande).

\section{Épandage des boues}

Les boues de consistance pâteuse ( $17 \%$ de MS) ont été épandues manuellement à la surface des parcelles en juillet 1986. Treize jours après l'épandage, elles ont été mélangées avec une fraise aux 20 premiers $\mathrm{cm}$ du sol. Le travail du sol a été aussi effectué dans le témoin sans apport.

\section{Prélèvements et traitement des échantillons de sols}

Les prélèvements ont été réalisés à la bêche dans la couche $0-20 \mathrm{~cm}$. L'échantillon moyen représentatif de chaque traitement est formé à partir de 4 prélèvements d'environ $1,5 \mathrm{~kg}$ (sol séché à l'air). Une séparation manuelle préalable des mottes de boues de taille supérieure à $5 \mathrm{~mm}$ de diamètre est effectuée. On obtient ainsi une fraction "boue" et une fraction "sol». Dans cette dernière, des agrégats de boue sont vi-

Tableau II. Principales caractéristiques chimiques des boues résiduaires (\% de boues séchées à $\left.105^{\circ} \mathrm{C}\right) . \mathrm{P}_{\mathrm{t}}$ : phosphore total; $\mathrm{P}_{\mathrm{m}}$ : phosphore minéral soluble dans $\mathrm{H}_{2} \mathrm{SO}_{4} 2 \mathrm{~N}$; $\mathrm{P}$ : phosphore organique; $\mathrm{P}_{\mathrm{NaHCO}_{3}}{ }^{-\mathrm{F}}$ : phosphore extrait dans $0,5 \mathrm{M} \mathrm{NaHCO}_{3}+0,5 \mathrm{M} \mathrm{NH}_{4} \mathrm{~F}$.

\begin{tabular}{|c|c|}
\hline Carbone \% & 38,9 \\
\hline Azote $\%$ & 6,8 \\
\hline $\mathrm{C} / \mathrm{N}$ & 6 \\
\hline N-NH4 \% & 0,065 \\
\hline $\mathrm{N}-\mathrm{NO} 3 \%$ & 0,188 \\
\hline $\mathrm{pH}$ eau & 6,2 \\
\hline \multicolumn{2}{|c|}{ Phosphore \% } \\
\hline $\mathrm{Pt}$ & 2,45 \\
\hline $\mathrm{Pm}$ & 0,82 \\
\hline Po & 0,96 \\
\hline $\mathrm{PNaHCO}_{3}-\mathrm{F}$ & 0,51 \\
\hline $\mathrm{Po} / \mathrm{Pm}$ & 1,2 \\
\hline $\mathrm{C} / \mathrm{Po}$ & 40 \\
\hline
\end{tabular}

sibles mais difficilement triables, leur taille étant essentiellement inférieure à $2 \mathrm{~mm}$. La fréquence des prélèvements est d'abord hebdomadaire, puis bimensuelle, bimestrielle et enfin trimestrielle.

\section{Caractérisation du phosphore}

Le phosphore total $\left(P_{t}\right)$ est déterminé après calcination $\left(550^{\circ} \mathrm{C}\right)$ de l'échantillon et repris par l'acide nitrique pur à chaud. Le phosphore organique $\left(P_{0}\right)$ est obtenu par différence des 2 extraits à $\mathrm{l}^{\prime} \mathrm{H}_{2} \mathrm{SO}_{4} 2 \mathrm{~N}$, avant et après calcination (Saunders et Williams (1955) modifié par Anderson, 1960). Le phosphore minéral $\left(P_{m}\right)$ est ici le phosphore extrait à froid par l' $\mathrm{H}_{2} \mathrm{SO}_{4} 2 \mathrm{~N}$ (17 h) lors de la détermination de $\mathrm{P}_{\mathrm{o}}$. Nous avons également déterminé la teneur en phosphore des extraits bicarbonate-fluorure d'ammonium $\mathrm{pH} 8,5$ selon Dabin (1967), cette forme est notée

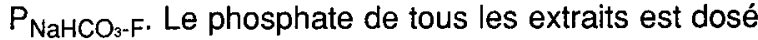
par colorimétrie (Duval, 1962), après complexation de l'ion fluor par l'acide borique dans le cas de $\mathrm{P}_{\mathrm{NaHCO}_{3}-\mathrm{F}}$. La précision des déterminations est de $3 \%$. Le calcul du rapport $C / P_{0}$ est fait après dosage du carbone par voie sèche (auto-analyseur CNS NA 1500 Carlo Erba).

\section{RÉSULTATS}

\section{États des microparcelles avant épandage et évolution du phosphore dans le sol témoin (tableau III)}

\section{Teneur en phosphore de l'horizon 0-20 cm des microparcelles avant épandage}

Les teneurs en $P_{m}$ et $P_{t}$ des prélèvements réalisés avant épandage dans les parcelles qui recevront ultérieurement 10 et $100 \mathrm{t}$ de boues sont inférieures à celles de la parcelle $(T)$. Les teneurs des autres formes du phosphore $\left(P_{0}\right.$ et $\mathrm{P}_{\mathrm{NaHCO}_{3}-\mathrm{F}}$ ) ne sont pas significativement différentes dans les 3 parcelles. Le rapport $\mathrm{C} / \mathrm{P}_{0}$ est le même dans les 3 parcelles : 77,75 et 75 pour $(T),(10 \mathrm{t})$ et $(100 \mathrm{t})$ respectivement. Le rapport $\mathrm{P}_{\mathrm{o}} / \mathrm{P}_{\mathrm{m}}$ moyen de l'horizon $0-20 \mathrm{~cm}$ de la parcelle témoin est de 1,7 avec un coefficient de variation de $7,3 \%$. Avant épandage de l'amendement ce rapport est de 1,9 et 2,1 pour les parcelles (10 t) et (100 t).

L'hétérogénéité initiale du phosphore total altère peu l'interprétation des résultats puisqu'il est démontré qu'il n'y a pas de rapport entre la teneur en phosphore total d'un sol et le phosphore assimilé par les plantes (Roche et al, 1980). 
Tableau III. Formes du phosphore $\left(\mu \mathrm{g} \mathrm{g}^{-1} \mathrm{sol}\right)$ des échantillons de l'horizon 0-20 $\mathrm{cm}$ des parcelles témoin [T], [10 t] et [100 t] avant épandage des boues, et teneurs moyennes des différentes formes du phosphore du sol témoin durant l'expérimentation. Abréviations : voir tableau II.

\begin{tabular}{ccccccc}
\hline Parcelles & jour * & $\mathrm{Pt}$ & $\mathrm{Pm}$ & $\mathrm{Po}$ & $\mathrm{PNaHCO}_{3}-\mathrm{F}$ & $\mathrm{C} / \mathrm{Po}$ \\
\hline$[\mathrm{T}]$ & -7 & 538 & 153 & 263 & 31 & 83 \\
& moyenne de -7 a 512 & 532 & 154 & 279 & 36 & 77 \\
& $\mathrm{CV} \%$ & 3,2 & 5,4 & 4,5 & 9,6 & 6,3 \\
{$[10 \mathrm{t}]$} & -7 & 470 & 142 & 268 & 28 & 75 \\
{$[100 \mathrm{t}]$} & -7 & 409 & 131 & 269 & 34 & 75 \\
\hline
\end{tabular}

*Après épandage des boues.

\section{Évolution du phosphore du sol témoin}

La teneur moyenne en phosphore total des prélèvements successifs est de $532 \mu \mathrm{g} \cdot \mathrm{g}^{-1}$ de sol. Le coefficient de variation des teneurs est de $3,2 \%$. Le phosphore organique et le phosphore minéral représentent en moyenne, respectivement, 52,4 et $28,5 \%$ du phosphore total. Les variations des formes totale, organique et minérale sont faibles et voisines de la précision de la mesure.

Les teneurs du $\mathrm{P}_{\mathrm{NaHCO}_{3}-\mathrm{F}}$ sont comprises entre 29 et $34 \mu \mathrm{g}(\mathrm{P}) \cdot \mathrm{g}^{-1}$ (de sol). Les variations de ces teneurs représentent $9,6 \%$ de la moyenne observée.

\section{Évolution de la masse et de la teneur en phosphore total de la fraction boue}

\section{Cinétique de disparition des mottes de boue}

La figure 1 montre l'évolution de la masse de matière sèche des mottes de boues triées dans les échantillons provenant des parcelles (10 t) et $(100 \mathrm{t})$. Cent jours après l'enfouissement, il n'est plus possible d'isoler des mottes de boues supérieures à $5 \mathrm{~mm}$ dans la parcelle $(10 \mathrm{t})$. Dans la

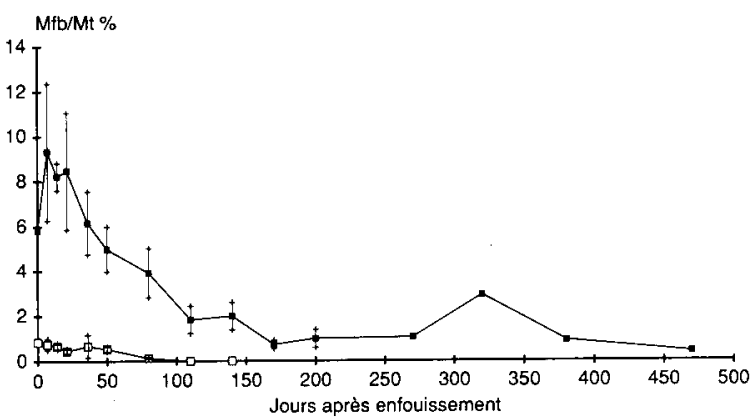

Fig 1. Évolution de la masse de matière sèche de la fraction boues $>5 \mathrm{~mm}$ (Mfb) par rapport à la masse de matière sèche totale $(\mathrm{Mt})$ du prélèvement parcelles $[10 \mathrm{t}]:(\square)$ et $[100 \mathrm{t}]:(\mathbb{\square}) ;$ écart type : $(\uparrow)$. parcelle (100 t) à l'inverse, les mottes de boues ne sont toujours pas totalement incorporées au sol après une année.

\section{Évolution de la teneur en phosphore total de la fraction boue (tableau IV)}

La teneur en phosphore total de la fraction boue tend à diminuer significativement $50 \mathrm{j}$ après l'enfouissement dans la parcelle $(100 \mathrm{t})$, et reste constante dans la parcelle (10 t). La quantité de $P_{t}$ des boues encore présentes dans le sol se déduit de la masse et la teneur du $P_{t}$ de la fraction boue. Dans la parcelle (10 t) la quantité de phosphore total contenu dans les agrégats de boues est de l'ordre de $100 \mu \mathrm{g} \mathrm{g}^{-1}$. Dans la parcelle $(100 \mathrm{t})$, la fraction boue représente à $21 \mathrm{j}$ un contenu de phosphore de $1847 \mu \mathrm{g} \cdot \mathrm{g}^{-1}$ de sol. Puis il diminue pour atteindre la valeur de 185 $\mu \mathrm{g} \cdot \mathrm{g}^{-1} 5$ mois après enfouissement. Cette fraction boue représente alors $8 \%$ du $P_{t}$ de l'horizon 0-20 cm étudié.

\section{Évolution des quatre formes du phosphore du sol dans les parcelles (10 t) et(100 t)}

\section{Traitement $(10 t)$}

Soixante jours après l'épandage, l'apport de $10 \mathrm{t}$ de boues sèches par ha induit une augmentation significative de la teneur en phosphore total. Le gain est de $100 \mu \mathrm{g}^{\circ} \mathrm{g}^{-1}$ en fin d'expérimentation (fig 2). Les variations observées concernent :

- le phosphore minéral dont la teneur a augmenté de $76 \mu \mathrm{g}^{\circ} \mathrm{g}^{-1}$;

- le phosphore extrait par le mélange bicarbonate-fluorure dont la teneur passe de 35 à $50 \mu \mathrm{g} \cdot \mathrm{g}^{-1}$ un mois après l'épandage;

- le phosphore organique, dont l'augmentation de $21 \mu \mathrm{g} \cdot \mathrm{g}^{-1}$ est significative un an après épan- 
Tableau IV. Teneur et évolution pondérale du phosphore total de la fraction boue dans l'horizon 0-20 cm.

\begin{tabular}{llcccc}
\hline Pancelle & $\begin{array}{l}\text { Jours après } \\
\text { épandage }\end{array}$ & $\begin{array}{c}\text { masse } \\
\text { fraction boue } \\
\text { g MS \% sol }\end{array}$ & $\begin{array}{c}\text { teneur } \mathbf{P} \\
\text { fraction boue } \\
\text { mg P.g }{ }^{-1} \text { fr. }\end{array}$ & $\begin{array}{c}\mu g \text { P-boues. } \\
\text { sol }\end{array}$ & $\begin{array}{c}\text { Pt boues } \\
\text { \% Pt sol }\end{array}$ \\
\hline$[10 \mathrm{t}]$ & 13 avant enfouissement & & 20,45 & - & \\
& 14 enfouissement & 0,85 & 17,72 & 150 & 21 \\
& 21 & 0,75 & 20,42 & 153 & 22 \\
& 28 & 0,66 & 18,52 & 122 & 18 \\
& 35 & 0,45 & 18,38 & 83 & 13 \\
& 50 & 0,67 & 15,96 & 107 & 16 \\
& 64 & 0,53 & 19,24 & 102 & 15 \\
{$[100 \mathrm{t}]$} & 13 avant enfouissement & & 20,45 & - & \\
& 14 enfouissement & 5,8 & 19,61 & 1137 & 60 \\
& 21 & 9,31 & 19,84 & 1847 & 68 \\
& 28 & 8,2 & 18,28 & 1499 & 63 \\
& 50 & 6,14 & 12,15 & 746 & 40 \\
& 64 & 4,97 & 13,19 & 655 & 27 \\
& 94 & 3,9 & 11,45 & 446 & 24 \\
& 150 & 1,82 & 10,16 & 185 & 8 \\
\hline
\end{tabular}

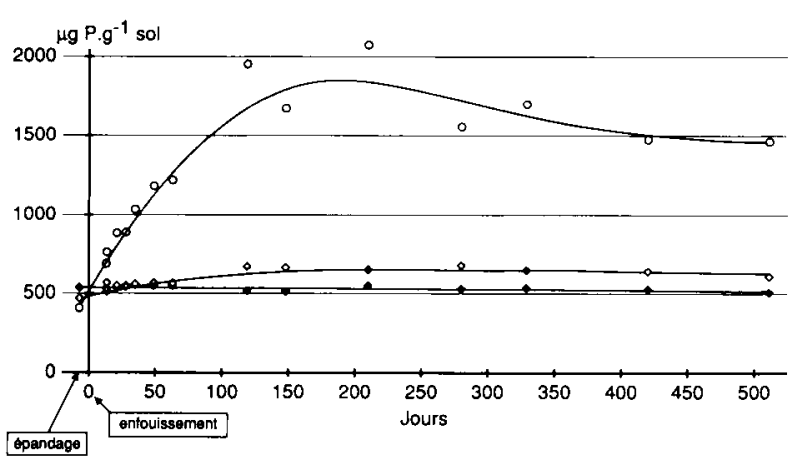

Fig 2. Évolution du phosphore total des parcelles témoin $(\triangleleft)$, $10 \mathrm{t}(\gg)$ et $100 \mathrm{t}(0)$.

dage, la différence avec le témoin étant de $30 \mu \mathrm{g} \cdot \mathrm{g}^{-1}$ (fig 3).

Ces variations conduisent à une modification de la proportion relative des différentes formes du phosphore. Le phosphore minéral croît de 30 à $38 \%$ du phosphore total, le phosphore extrait par un mélange bicarbonate-fluorure de 6 à $11 \%$, et, quoiqu'augmentant en valeur absolue, le phosphore organique diminue de 57 à $47 \%$. $100 \mathrm{j}$ après l'épandage, le rapport $P_{\mathrm{o}} / \mathrm{P}_{\mathrm{m}}$ initialement égal à 1,9 , est compris entre 1,4 et 1,7 . II est significativement inférieur à celui de la parcelle témoin. Après $270 \mathrm{j}$ d'observation le rapport $\mathrm{C} / \mathrm{P}_{\mathrm{o}}$ est inférieur à celui du traitement témoin.

\section{Traitement $(100 \mathrm{t})$}

L'apport de 100 t MS de boues par ha provoque un accroissement immédiat du phosphore total. En 13 jours, temps écoulé entre l'épandage et l'enfouissement, le phosphore total s'accroît de $68 \%$ dans la couche $0-20 \mathrm{~cm}$. La teneur en

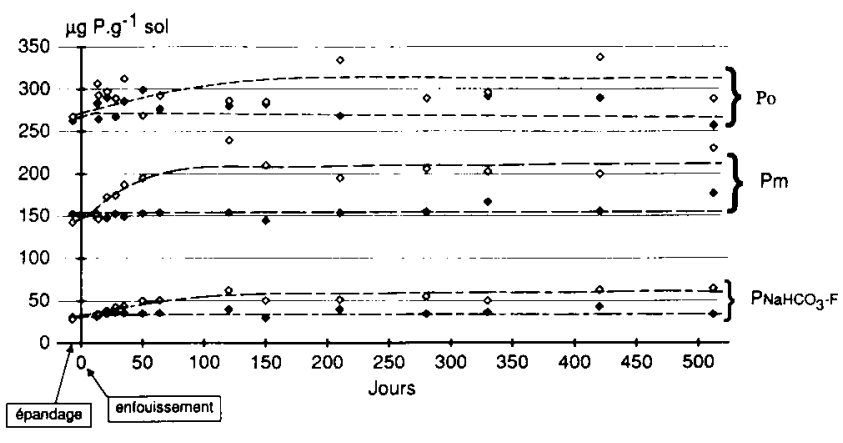

Fig 3. Évolution des formes du phosphore des traitements témoin $(\diamond)$ et $10 \mathrm{t}(\diamond)$ : organique $\left(P_{0}\right)$, minérale $\left(P_{m}\right)$ et extrait par le mélange bicarbonate-fluorure $\left(\mathrm{P}_{\mathrm{NaHCO}_{3}}-\mathrm{F}\right)$.

phosphore total dans le sol se stabilise à 1400 $\mu \mathrm{g} \cdot \mathrm{g}^{-1}$ en fin d'expérience (fig 2 ).

Les variations concernent :

- la teneur en $P_{m}$ qui atteint $765 \mu \mathrm{g} \cdot \mathrm{g}^{-1}$, soit 6 fois la teneur initiale observée, et 5 fois la teneur moyenne du témoin;

- le phosphore extrait par le mélange bicarbonate-fluorure dont la teneur a décuplé pour atteindre $325 \mu \mathrm{g} \cdot \mathrm{g}^{-1}$;

- la teneur en $P_{o}$ qui suit une évolution similaire, elle atteint $409 \mu \mathrm{g} \cdot \mathrm{g}^{-1}$ en fin d'observation, soit une augmentation de $52 \%$ (fig 4 ).

À $500 \mathrm{j}$ la proportion des formes de phosphore organique et minéral du sol est modifiée : $\mathrm{P}_{\mathrm{m}}$ augmente 32 à $50 \%$ de $\mathrm{P}_{\mathrm{t}}$ en fin d'expérience, tandis que $P_{\circ}$ diminue de 66 à $28 \%$.

Après l'enfouissement des boues le rapport $\mathrm{C} /$ $P_{0}$, initialement égal à 75 , diminue jusqu'à 50 , il est égal à 65 en fin d'observation. Le rapport $P_{0} /$ $P_{m}$, initialement de 2,1 décroît et reste compris entre 0,7 et 0,9 . 


\section{DISCUSSION}

La variabilité initiale du contenu en phosphore des parcelles est imputable aux nombreuses années de fertilisation. Les stocks de phosphore observés ne peuvent s'expliquer que par le caractère fortement fixateur de ces sols ferrallitiques à l'égard du phosphore (Brossard et al, 1988).

Les boues utilisées correspondent à un instant de 245 et de $2450 \mathrm{~kg} \cdot \mathrm{ha}^{-1}$ de phosphore total respectivement pour les parcelles $(10 \mathrm{t})$ et $(100 \mathrm{t})$, qui contiennent initialement 846 et 736 $\mathrm{kg} \cdot$ ha $^{-1}$ dans l'horizon $0-20 \mathrm{~cm}$ ( $\delta a$ égale à 0,9 ). Cinq cent jours après l'apport au sol, le bilan est égal à 101 et $78 \%$ des apports. Pour la parcelle (100 t) les 22\% manquants, qui représentent 700 $\mathrm{kg} \cdot \mathrm{ha}^{-1}$, doivent être recherchés dans les horizons inférieurs à $20 \mathrm{~cm}$ de profondeur, où un transfert de phosphore en solution est possible. La teneur en phosphore de la couche $0-20 \mathrm{~cm}$ a de fait augmenté de $502 \mathrm{~kg} \cdot \mathrm{ha}^{-1}$ entre l'épandage et l'enfouissement des boues. Cette augmentation pourraît être provoquée par le flux d'eau de percolation (pluviométrie de $81 \mathrm{~mm}$ durant cette période). La concentration moyenne de phosphore dans les eaux de percolation aurait été de $620 \mathrm{mg} \cdot \mathrm{l}^{-1}$. Cette valeur est du même ordre de concentration du phosphore dans un mélange boue-eau effectué par ailleurs qui atteint $444 \pm 58 \mathrm{mg}$ (Monteau, 1987).

La teneur en phosphore total dans les boues utilisées $(2,45 \%)$ est comparable aux teneurs de boues urbaines obtenues par ailleurs avec les mêmes technologies (Sommers, 1977; Morel, 1978; De Haan, 1980). Comparées aux composts disponibles aux Antilles, leur teneur en phosphore total est supérieure : $0,2 \%$ pour les composts d'ordures ménagères (Clairon et al,

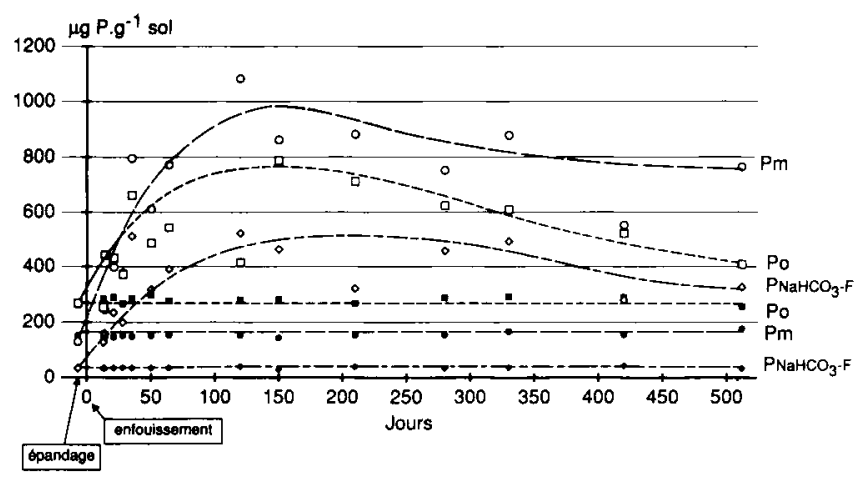

Fig 4. Évolution des formes du phosphore des traitements témoin (symboles noirs) et $100 \mathrm{t}$ (symboles blancs) : organique $\left(P_{o}\right)$, minérale $\left(P_{m}\right)$ et extrait par le mélange bicarbonate-fluorure $\left(\mathrm{P}_{\mathrm{NaHCO}_{3}}{ }^{-} \mathrm{F}\right)$..
1982); 0,13\% pour les bagasses compostées (Action Cordet, 1981). Les boues étudiées ont un apport $P_{o} / P_{m}$ de 1,2. Dans les boues urbaines, la teneur en phosphore minéral est généralement supérieure à celle en phosphore organique : Sommers et al (1976) indiquent que 64 à $84 \%$ du phosphore total sont solubles dans $\mathrm{HCl} 1 \mathrm{~N}$. Le phosphore minéral des boues peut être adsorbé sur les oxyhydroxydes de fer et d'aluminium, et ou lié à des cations comme le calcium (Gupta, 1976). Les boues utilisées ont été floculées en labsence de calcium et de fer, ce qui explique qu'elles ne présentent pas de pouvoir fixateur à l'égard des ions phosphate (Monteau, 1987). Ces boues ont une teneur en phosphore organique égale à $0,96 \%$. La minéralisation nette du phosphore organique a été démontrée sur des cultures de microorganismes et des composts de résidus végétaux pour des teneurs en phosphore organique supérieures à 0,3\% (Anderson, 1975). Ainsi, caractéristiques chimiques et pouvoir tampon à l'égard du phosphore indiquent que ces boues sont aptes à fournir du phosphore.

Le transfert de phosphore depuis les boues jusqu'au sol est continu dans la parcelle (10 t) durant les 3 premiers mois de l'expérimentation. La désagrégation des mottes de boues (fraction boues $>5 \mathrm{~mm}$ ) s'accompagne d'une augmentation de la teneur en $\mathrm{P}_{\mathrm{t}}$ de la fraction sol. En incubations contrôlées, Monteau (1987) a montré que la concentration du phosphore dans la solution d'un mélange eau-boue évoluait de 125 à $400 \mathrm{mg} \cdot \mathrm{l}^{-1}$ après $20 \mathrm{j}$ d'incubation. Les caractéristiques chimiques des boues expliquent l'accroissement des teneurs en phosphore extrait par un mélange bicarbonate-fluorure $\left(\mathrm{P}_{\mathrm{NaHCO}_{3}}\right.$ F) et par $\mathrm{H}_{2} \mathrm{SO}_{4} 2 \mathrm{~N}\left(\mathrm{P}_{\mathrm{m}}\right)$ de la fraction sol. Après $500 \mathrm{j}$ le rapport $\mathrm{P}_{\mathrm{o}} / \mathrm{P}_{\mathrm{m}}$ du sol a significativement diminué dans les 2 traitements : de 1,9 à 1,7 pour la parcelle $(10 \mathrm{t})$, de 2,1 à 0,9 pour la parcelle (100 t). L'accroissement de $P_{m}$ serait dû au passage dans le sol d'ions phosphate contenus dans les corps microbiens dont les parois seraient lysées au cours des processus de dégradation. Les parcelles étudiées ont un rapport $\mathrm{C} / \mathrm{P}_{\mathrm{o}}$ égal à 75 . Si l'interprétation de cette donnée est souvent controversée (Dommergues et Mangenot, 1970; Dalal, 1977), de telles valeurs sont considérées comme étant favorables à la minéralisation du phosphore organique. Les boues sont un fort intrant de carbone avec un rapport $\mathrm{C} / \mathrm{P}_{\mathrm{o}}=40$. Cinq cent jours après l'enfouissement des boues ce rapport est de 74 dans la parcelle $(10 t)$ et de 65 dans la parcelle $(100 \mathrm{t})$. Dans la parcelle $(100 \mathrm{t})$ une telle valeur traduit la persistence de fines particules de boue. 
II n'est pas possible de quantifier avec précision la part des transferts de phosphore en solution et celles des transferts sous forme figurée (agrégats de boues de taille inférieure à $5 \mathrm{~mm}$ ). Le rôle de la faune du sol dans le mélange peut aussi être envisagé (Kobel-Lamparski et Lamparski, 1987). On peut penser que la fraction sol est constituée d'une juxtaposition d'agrégats de sol et de particules de boue. Ceci pourraît être confirmé par une étude, actuellement en cours, de la matière organique et du phosphore, après fractionnement granulométrique des sols.

D'un point de vue agronomique, l'accroissement de la teneur en phosphore du sol doit entraîner une augmentation de la mise à disposition d'ions phosphate pour la plante. Lors de l'apport de $10 \mathrm{t}$ de boues la concentration en phosphore de l'extrait bicarbonate-fluorure augmente puis reste relativement constante $50 \mathrm{j}$ après l'enfouissement. Le mélange bicarbonate-fluorure met en solution une partie des phosphates liés au fer, au calcium et à l'aluminium. Morel et Fardeau (1987) ont montré que le phosphore de ce type d'extrait est en grande partie sous une forme non isotopiquement échangeable, donc non assimilable à court terme. En incubation, l'apport de $100 \mathrm{t}$ de boues provoque des modifications positives du pool de phosphate assimilable, alors que l'apport de 10 t de boues n'augmente significativement la concentration en phosphate de la solution (mélange eau-sol) que temporairement, entre le $10^{\mathrm{e}}$ et le $21^{\mathrm{e}} \mathrm{j}$ d'incubation (Monteau, 1987). Comparé à une fertilisation équivalente en superphosphate, l'apport de boues du même type entraîne une augmentation significative de la teneur en phosphore des parties aériennes du maiis, mais non des grains (Giboulot, 1984). Depuis une quinzaine d'années aux Petites Antilles de systèmes de culture intensifs à cycles courts sont développés et induisent à terme la baisse des réserves de matière organique avec modification des propriétés physiques et chimiques de sols ferrallitiques et de vertisols (Feller et al, 1990). Dans de telles situations, des apports massifs de boues (MS) de 100 tha $^{-1}$ seraient justifiés pour rétablir les réserves organiques et minérales du sol.

\section{CONCLUSION}

Les boues de station d'épuration urbaine peuvent constituer un amendement phosphaté intéressant à valoriser en milieu tropical humide. L'étude de l'évolution de formes du phosphore dans un sol ferrallitique, maintenu sans végétation, montre qu'après l'épandage et l'enfouissement des boues:

- la teneur en phosphore total du sol a nettement augmenté, et les formes organiques et minérales du phosphore sont concernées par cette augmentation;

- le lessivage du phosphore minéral et/ou le transfert de la boue sous forme particulaire fine vers le sol peut être très rapide et provoque l'accroissement des teneurs en phosphore extrait par un mélange bicarbonate-fluorure et phosphore acido-soluble;

- l'effet sur l'augmentation de la teneur en phosphore extrait par un mélange bicarbonatefluorure se maintient au delà de 12 mois.

En agriculture l'utilisation de ces boues (MS) à des doses voisines de 10 th ha $^{-1} \mathrm{a}$ un effet à court et moyen terme sur la fourniture en ions phosphate au sol, cette dose équivaut à une forte fumure phosphatée. Les apports massifs, comme le montre la dose de $100 \mathrm{t}^{\cdot h \mathrm{a}^{-1}}$, peuvent se justifier en zone tropicale afin d'accroître ou de maintenir la productivité de sols dégradés par des cultures continues à faibles restitutions organiques. Toutefois, il faut dans ce cas tenir compte d'autres paramètres comme la production de nitrates, les risques d'acidification du sol et de pollution par les micropolluants.

\section{RÉFÉRENCES}

Action Cordet (1981) Recyclage de la matière organique dans les sols. Rapport final de Convention Orstom-INRA-IRAT, Orstom-Martinique, $27 \mathrm{p}$

Anderson $G$ (1960) Factors affecting the estimation of phosphate-esters in soils. $J$ Sci Food Agric 11, 497-503

Anderson G (1975) Other organic compounds. In: Soil Components. Vol I. Organic Components (JE Gieseking, ed) Springer-Verlag, New York, 305-331

Brossard M, Fardeau JC, Monteau JP, Laurent JY (1988) Matière organique et mobilité du phosphore dans quelques types de sols. Rapport Convention CEE-Orstom «Fertilité des sols dans les agricultures paysannes caribéennes", Orstom-Martinique, 69-84

CPCS (1967) Classification des sols. École nationale supérieure agronomique Grignon, $87 \mathrm{p}$

Chaminade R, Roche P, Grière L (1977) La carence en phosphore dans les sols tropicaux. CR Acad Agric Fr 6, 479-482

Chevignard T, Fardeau JC, Doulbeau-Piasco S, Feller C, Turenne JF, Vallerie M (1986) Effet du remodelage parcellaire sur la fixation des phosphates en 
divers types de sols des Antilles. Agronomie 6, 149-156

Clairon M, Zinsou C, Nagou D (1982) Étude des possibilités d'utilisation agronomique des composts d'ordures ménagères en milieu tropical. 1. Compostage des ordures ménagères. Agronomie 2, 295300

Clairon M, Giboulot MC, Sobesky O (1989) Effets d'apports de boues activées sur maïs et sur sol ferrallitique. $25^{\circ}$ Congrès CFCS, $2-8$ juillet 1989 , Guadeloupe

Dabin B (1963) Appréciation des besoins en phosphore dans les sols tropicaux. Les formes du phosphore dans les sols de Côte d'Ivoire. Cah Orstom Sér Pédol 1, 27-42

Dabin B (1967) Application des dosages automatiques à l'analyse des sols. 3. Cah Orstom Sér Pédol $5,257-286$

Dalal RC (1977) Soil organic phosphorus. In: Advances in Agronomy. Vol 29 (NC Brady, ed) Acad Press, Londres, 83-117

De Hann S (1980) Boues d'épuration comme engrais phosphatés. Phosphorus Agric 78, 37-44

Dommergues $Y$, Mangenot F (1970) Écologie microbienne du sol. Masson, Paris

Duval L (1962) Dosage céruléomolybdique de l'acide phosphorique dans les sols, les végétaux et les engrais. Ann Agron 469-482

Feller C, Albrecht A, Brossard M, Chotte JL, Cadet P, Hétier JM, Barrois I, Mariotti A, Castellanet C, Guiran $E$ de, Clairon $M$, Daly $P$, Mahieu M, Pilgrim $R$, Ramdass A, Ahmad N, Griffith SM, Fardeau JC (1990) Effets de différents systèmes de culture sur quelques propriétés des sols et relations sol-plante dans la zone des Petites Antilles. In: Agricultures paysannes et développement. Sacad, Univ AntillesGuyane, 3, 162-190

Fox RL, Plucknette DL, Whitney AD (1968) Phosphate requirements of Hawaï latosols and residual effects of fertilizers phosphorus. 9th Int Congr Soil Sci, Adelaïde Vol II, 301-310

Giboulot MC (1984) Effets d'apports de boues résiduaires de station d'épuration en sol ferrallitique tropical: modifications révélées par le comportement d'un peuplement de maïs. Mémoire, Ina Paris-Grignon, $53 p$

Gupta SK (1976) Phosphate removal in systems $\mathrm{H}_{2} \mathrm{PO}_{4}-\mathrm{FeO}(\mathrm{OH})$ and $\mathrm{H}_{3} \mathrm{PO}_{4}-\mathrm{FeCl}_{3}$ and characte- ristics of sludge phosphate. Thesis Fac of Natural Sci, Univ Berne $138 \mathrm{p}$

Hinesly TD, Jones RL, Ziegler EL (1973) Effects on corn by application of heated anaerobically digested sludges. Compost Sci 13, 26-30

King LD, Morris HD (1972) Land disposal of liquid sewage sludge. 1 . The effect on yield, in vivo digestibility and chemical composition of Coastal Bermuda grass (Cynodon dactylon). J Environ Qual 1, 325329

Kobel-Lamparski A, Lamparski F (1987) Effect of sludge on the structure of the upper soil layers and on the earthworms of a beech woodland: In: On earthworms (AM Bonvicini Pagliai, P Omodeo, eds) Selected Symposia and Monographs UZI, 2, Mucchi, Modena, 409-417

Mench M, Clairon M, Sobesky O, Nagou D (1989) Dynamique à court terme de l'azote minéral en sol ferrallitique nu après apport d'une boue urbaine. Agronomie $9,785-793$

Monteau JP (1987) Devenir in vitro d'amendements organiques dans deux sols tropicaux acides. Effets sur la disponibilité du phosphore et sur la teneur en potassium soluble. Mém ISA-Lille, Cea-Orstom $43 p$

Morel Jl (1978) Boues résiduaires et fertilisation phosphatée. Phosphorus Agric 73, 15-22

Morel C, Fardeau JC (1987) Le phosphore assimilable des sols intertropicaux : ses relations avec le phosphore extrait par deux méthodes chimiques. Agron Trop 42, 248-257

Nagou D, Clairon M (1985) Et si ... l'agriculture guadeloupéenne recyclait les déchets ? Exemple des boues urbaines. Bull Agron Antilles-Guyane 3, 1-3

Roche $P$, Grière L, Babre $D$, Calba $H$, Fallavier $P$ (1980) Le phosphore dans les sols intertropicaux : appréciation des niveaux de carence et des besoins en phosphore. IMPHOS Publ Sci $n^{\circ} 2$, Paris, $48 \mathrm{p}$

Saunders WMH, Williams EG (1955) Observations on the determination of total organic phosphorus in soils. J soil Sci 6, 254-267

Sommers LE (1977) Chemical composition of sewage sludge and analysis of their potential use as fertilizers. J Environ Qual 6, 225-232

Sommers LE, Nelson DW, Yost KJ (1976) Variable nature of chemical composition of sewage sludges. J Environ Qual 5, 303-306 